\title{
The Soret Effect in Naturally Propagating, Premixed, Lean, Hydrogen-Air Flames
}

\author{
Joseph F. Grcar $\quad$ John B. Bell ${ }^{\mathrm{a}} \quad$ Marcus S. Day $^{\mathrm{a}}$ \\ ${ }^{a}$ Center for Computational Science and Engineering \\ Lawrence Berkeley National Laboratory \\ Berkeley, CA 94720-8142, USA
}

\begin{abstract}
Comparatively little attention has been given to multicomponent diffusion effects in lean hydrogen-air flames, in spite of the importance of these flames in safety and their potential importance to future energy technologies. Prior direct numerical simulations either have considered only the mixture-averaged transport model, or have been limited to stabilized flames that do not exhibit the thermo-diffusive instability. The so-called full, multicomponent transport model with cross-diffusion is found to predict hotter, significantly faster flames with much faster extinction and division of cellular structures.
\end{abstract}




\section{Nomenclature}

$\mathcal{D}_{k, \ell}$ binary diffusion coefficient

$D_{k, \ell}$ species diffusion coefficient

$D_{k}^{\operatorname{mix}}$ species mixture-averaged diffusion coefficient

$\theta_{k}$ species thermal diffusion coefficient

$\delta_{T}$ thermal flame thickness

$h_{k}$ specific enthalpy of the species

$\mathbf{j}_{k}^{(i)}$ species diffusive flux vector, the superscript $i=0,1,2$ indicating the degree of approximation

$\mathbf{j}_{k}^{\text {cor }}$ corrected flux, derived from $\mathbf{j}_{k}^{(i)}$ for $i=1,2$

$\kappa$ curvature of the flame surface (positive when the flame is convex toward the fresh mixture)

$k, \ell$ species indices

$\mathcal{L}$ Markstein length

$\lambda$ thermal conductivity (of the mixture)

$\lambda^{\prime}$ partial thermal conductivity

$p$ pressure

q heat flux vector

$\rho$ mass density

$\rho_{k}$ species mass density, $\rho Y_{k}$ 
$s$ local burning velocity

$s_{0}$ burning velocity of the planar flame

$S$ strain tangential to the flame surface

$T$ temperature

$X_{k}$ species mole fraction

$Y_{k}$ species mass fraction

\section{Introduction}

There are compelling reasons to develop models that faithfully reproduce the thermo-diffusive instability of lean premixed, hydrogen-air flames. These flames may someday power turbines for electric power generation [1], and they have long been recognized as potential dangers in nuclear reactor accidents [2]. Hydrogen can also be used to increase the flammability of other fuels under lean conditions [3]. Lean hydrogen flames differ from lean hydrocarbon flames because the highly diffusive fuel causes them to exhibit the thermo-diffusive instability. Other fuels blended with hydrogen inherit the instability [4].

Predictions of extinction strain rates and laminar flame speeds agree well with experiment across the whole range of lean to rich hydrogen-air mixtures $[5,6,7]$, but when the focus is just very lean flames, then the relative scatter is large. The variation can be seen for extinction strain rates in [6, Fig. 4], and for flame speeds in Fig. 1 of the present paper. It is tempting to believe chemical kinetics may account for most of the variation. For example, the large sensitivities of hydrogen-air, laminar flame speeds to reaction rates in GRI-Mech-2.11 are given in [5]. Nevertheless, predictions are comparably sensitive to both reaction and transport properties $[8,6,9,10,11]$.

Much less attention has been given to the influence of the diffusive transport terms in the reacting flow equations. For nonpremixed, methane-air, counterflow flames, Williams [11] found that extinction strain rates vary by $20 \%$ 
when predicted by different transport models. For premixed, hydrogen-air, flat flames, Bongers and de Goey [12] found different transport models had a large effect on predicted speeds in rich mixtures, but "for low values of the fuel equivalence ratio, $\phi$, the choice of the diffusion model is insignificant." Further, the Soret effect can be important, corroborating an early study of rich flames by Greenberg [13], but for low values of $\phi$ the effect is negligible. For example, with $\phi=0.37$ under atmospheric conditions, the speed calculated by $[14,15]$ is $15.52 \mathrm{~cm} / \mathrm{s}$ for a mixture model, and is $15.49 \mathrm{~cm} / \mathrm{s}$ for a multicomponent model including the Soret effect. For premixed, lean, hydrogen-air, conical (Bunsen) flames, Ern and Giovangigli [16] found "when thermal diffusion is included, active radicals are less prone to diffuse into the cold region and the flame propagation velocity is lower." Their explanation for the lowered speeds of flames convex to the fresh mixture would also pertain to the flat flames studied by Bongers and de Goey.

This paper considers lean, premixed, hydrogen-air flames that propagate freely and are therefore nonplanar and unsteady. Comparisons are made between the mixture-averaged diffusion model, and the multicomponent model with Dufour and Soret effects; the Soret effect is most relevant for the flames of interest. Previous computational studies of this type of flame, [17, 18, 19, 20,21, 22], have neglected thermal diffusion. Photographs of laminar flames [4, 23] and planar laser induced fluorescence imaging of turbulent flames [18] indicate the natural shape of these fames consists of cellular structures that are convex to the fresh gas. For these cellular flames, including Soret effects increases the predicted flame speed by $20 \%$ globally and by up to $170 \%$ locally, in contrast to the planar case of Bongers and de Goey and to the conical case of Ern and Giovangigli. Of significance for flamelet models is that the local acceleration of the flame is found not to satisfy a linear Markstein rule. Moreover, the two transport models predict qualitatively different cellular dynamics. 


\section{Transport Model Summary}

The transport terms enter into the conservation equations for reacting gases through divergences of fluxes. See, for example, [24] for derivation and discussion. The species and heat flux vectors have the general forms

$$
\begin{aligned}
\mathbf{j}_{k}^{(0)} & =\rho_{k}\left(-\sum_{\ell} D_{k, \ell} \mathbf{d}_{\ell}-\frac{\theta_{k}}{T} \nabla T\right) \\
\mathbf{q} & =\sum_{k} h_{k} \mathbf{j}_{k}-\lambda^{\prime} \nabla T-p \sum_{k} \theta_{k} \mathbf{d}_{k}
\end{aligned}
$$

where in the absence of species-specific body forces,

$$
\mathbf{d}_{k}=\nabla X_{k}+\left(X_{k}-Y_{k}\right) \frac{\nabla p}{p}
$$

The last term in Eqn. (1) is the Ludwig-Soret effect, also known as thermal diffusion. The last term in Eqn. (2) is the Dufour effect. They are both known as "cross-diffusion" terms. Equations (1-3) are collectively called "multicomponent diffusion with Dufour and Soret effects."

These equations permit some simplification. The spatial fluctuation of $p$ is small for low Mach number flows, which is called the combustion or isobaric approximation, so Eqn. (3) becomes $\mathbf{d}_{k} \approx \nabla X_{k}$. Omitting the Soret term from Eqn. (1) and the definition of the $D_{k, \ell}$ gives the Stefan-Maxwell equations

$$
\nabla X_{k}=\sum_{\ell} \frac{X_{k} X_{\ell}}{\mathcal{D}_{k, \ell}}\left(\frac{\mathbf{j}_{\ell}^{(1)}}{\rho_{\ell}}-\frac{\mathbf{j}_{k}^{(1)}}{\rho_{k}}\right)
$$

From these with additional assumptions follow the trace-species approximation

$$
\mathbf{j}_{k}^{(1)}=\rho_{k}\left(-\frac{D_{k}^{\operatorname{mix}}}{X_{k}} \nabla X_{k}\right)
$$

This equation is reasonable for combustion in air where nitrogen is the majority species. Neglecting spatial fluctuations of the mean mass further simplifies the approximation to

$$
\mathbf{j}_{k}^{(2)}=-\rho D_{k}^{\operatorname{mix}} \nabla Y_{k}
$$

For derivation see [25]. Both Eqns. $(4,5)$ must be corrected to conserve mass, which is often done by the formula

$$
\mathbf{j}_{k}^{\text {cor }}=\mathbf{j}_{k}-Y_{k} \sum_{\ell} \mathbf{j}_{\ell}
$$


With the corresponding removal of the cross-diffusion term from the heat flux,

$$
\mathbf{q} \approx \sum_{k} h_{k} \mathbf{j}_{k}-\lambda \nabla T
$$

Eqns. (4 or 5) and $(6,7)$ are the "mixture-averaged diffusion" model.

The choice between Eqns. (1-3) or (5-7) is effectively whether or not to include the Soret effect. Removing that term allows the remainder of Eqn. (1) to be reasonably approximated by Eqn. (5) for flames in air. In premixed flames the gradients of reactants and products are opposed, making the Dufour effect small, which was observed by Bongers and de Goey [12]. Thus the omission of the Soret term is the principal unaccounted approximation made when deriving Eqns. (5-7) from (1-3). Dixon-Lewis et al. [26] and Warnatz [27] suggest that the mixture-averaged model supplemented with a Soret term for light species is satisfactory, but Rosner, Israel, and La Mantia [28] gave several quantitative examples where the Soret effect is also important for heavy species. In this paper, we refer to Eqns. (1-3) as the mixture-averaged model, and to Eqns. (5-7) as the multicomponent model. We attribute the differences between them to the inclusion of the Soret effect in the multicomponent model.

\section{Computational Study}

Here we test the difference between Eqns. (1-3) and (5-7) in freely-propagating lean, premixed, hydrogen-air flames. The simulations are based on the low Mach number algorithm, with adaptive mesh refinement, developed by Day and Bell in [29]. The low Mach number equations are well known and are written in full in the previous publication. Since this approach filters out acoustic waves, simulations can be economically undertaken for time periods that are comparatively long for direct numerical simulations.

The original algorithm is based the mixture-averaged model of Eqns. (5-7), discretized using a Crank-Nicolson approximation; see [29] for details. For the multicomponent model of Eqns. (1-3), the implicit formulation has been replaced by the Heun second-order explicit Runge-Kutta formula.

The present calculations use either Eqns. (1-3) or (5-7) for a hydrogen-air mixture with equivalence ratio $\phi=$ 
0.37. The domain is a $1 \times 2 \mathrm{~cm}$ channel with resolution 39 microns. The domain is periodic in $x$ with inflow at the bottom, outflow at the top. Transport properties are computed using the EGLIB software of Ern and Giovangigli [30, 31]. The chemical mechanism is the sub-mechanism of GRI-Mech 2.11 [14] consisting of $\mathrm{N}_{2}$ and species comprised only of $\mathrm{H}$ and $\mathrm{O}$ atoms. Gravity and radiative losses are neglected.

Initial conditions are an artificially wrinkled flame extending across the width of the channel. The flame is held approximately $0.75 \mathrm{~cm}$ above the bottom boundary by dynamically adjusting the inflow using the control method described in [32]. The duration of the separate calculations for the mixture-averaged and multicomponent models is 0.1 and 0.04 seconds, respectively. Subsequent statistical analysis is based on the simulations omitting the first 0.01 seconds to avoid initial transients.

The flame front rapidly breaks into disconnected flames that are convex with respect to the fresh gas, and are separated by narrow, extinguished regions, as seen in Fig. 2. Some of these cells extinguish as they compete for fuel, but division of the surviving cells replaces those lost. Markstein [4] and Mitani and Williams [23] report experimental observations of this cellular phenomenon.

\section{Results}

\subsection{Enhanced Flame Speed}

The multicomponent model results in significantly higher flame speeds than the mixture-averaged model. In the planar case, the PREMIX software predicts a burning velocity of $15.5 \mathrm{~cm} / \mathrm{s}$. As noted in [12], this planar value is independent of the transport model. For the freely propagating flame, the predicted speed averages $34.9 \mathrm{~cm} / \mathrm{s}$ for the mixture-averaged model, and $42.5 \mathrm{~cm} / \mathrm{s}$ for the multicomponent model.

The effect of the multicomponent model is stronger locally, as seen in Fig. 3. The mode of the probability distribution for local flame speed is $1.8 s_{0}$ with the mixture-averaged model, but $2.7 s_{0}$ with the multicomponent model. This enhanced local burning is solely responsible for the higher overall flame speed, because the flame area 
is actually smaller. The time-averaged length of the flame is $1.27 \mathrm{~cm}$ for the mixture-averaged model, but $1.22 \mathrm{~cm}$ for the multicomponent model. Flame length is measured by using a surrogate for flame position consisting of the $1144 \mathrm{~K}$ isotherm (visible in Fig. 2) when it is convex to the fresh mixture where most burning occurs.

The local flame speeds in Fig. 3 are consumption speeds calculated by the method described in [32]. Briefly, the flame location is chosen to be the $1144 \mathrm{~K}$ isotherm. Since the flame is convex toward reactants but the isotherm is concave when passing through extinguished regions, the analysis is restricted to convex portions of the isotherm. That is, the analysis is "conditioned" by positive curvature. For these portions of the isotherm, fuel consumption is integrated over narrow strips 25 microns wide which extend $1 \mathrm{~mm}$ to either side of the $1144 \mathrm{~K}$ isotherm and whose sides follow the gradient of temperature (that is, the strips are perpendicular to every isotherm crossed). The integrated consumption is converted to a velocity by dividing it by the width of the strip and by the mass density of fuel in the fresh mixture.

\subsection{Unchanged Reaction Network}

The higher flame speeds predicted with the multicomponent model are not caused by changes to the chemical reactions pathways in the flame. Figure 4 shows spatially-integrated reaction rates of progress for the two simulations, normalized by the dominant reaction, for the states pictured in Fig. 2. There is little or no difference between the the reaction pathways for the two models. Similar results, not shown, were obtained when the integration was restricted to the small, rectangular regions visible in Fig. 2.

Figure 4 also depicts the reaction network for hydrogen. This network is prepared by decomposing the reactions to indicate the moles of hydrogen moved among the various species as determined by the integrated reaction rates. Since the latter are the same for both transport models, so is the network. 


\subsection{Higher Effective Equivalence Ratio}

Sivashinsky [33] noted that Zeldovich [34] gave the first qualitative description of the thermo-diffusive instability. When the deficient reactant is more diffusive than heat, then any portions of the flame protruding into the fresh mixture burn more vigorously because diffusive focusing of reactants into the flame is more rapid than heat diffusion away from the bulging front. This phenomena is demonstrated here by Fig. 5, which displays the joint probability distribution (JPDF) of $X\left(\mathrm{H}_{2}\right)$ and temperature. To avoid the larger pre- and post-flame regions from dominating the distribution, the JPDF is constructed using the method of conditional percentiles [35]. Each vertical slice through Fig. 5 is a separate probability density for $X\left(\mathrm{H}_{2}\right)$ at the given temperature. Additionally, Fig. 5 shows actual probability in terms of percentiles of the distribution. The color scale marks the percentiles of the probability density in order from lowest density (grey and blue) to highest (red and black).

The greater enhancement of combustion by the multicomponent model can be quantified by examining the distribution of hydrogen through the flame zone. The solid and dashed lines overlaid on Fig. 5 are for planar flames computed by PREMIX for various fuel equivalence ratios, $\phi$. The preponderance of density percentiles above the red solid lines in Fig. 5 shows the freely propagating flame, with either transport model, tends to have greater $X\left(\mathrm{H}_{2}\right)$ than the planar flame of the same $\phi=0.37$. With the mixture-averaged model, the distribution of $X\left(\mathrm{H}_{2}\right)$ tends to that of a planar flame with $\phi=0.41$ (white dashed line on left). With the multicomponent model, it tends to a still richer planar flame with $\phi=0.44$ (dashed line on right). Thus the mixture-averaged model significantly under-predicts the flux of fuel into the cellular structures.

\subsection{Compounded Soret Effect}

The Soret effect plays a crucial role in the difference in burning between the mixture model and the multicomponent model. The thermal diffusion coefficient for molecular hydrogen is negative, so thermal diffusion supplements the diffusive flux of fuel into the reaction zone. This enhanced transport further increases the effective richness of the

mixture, with concomitant increase to the temperature gradient, so that the Soret effect is compounded. The net 
effect of this compounding leads to peak values of the magnitude of $|\nabla T| / T$ of $4.1: 3.4: 1.7 / \mathrm{mm}$, respectively, in the freely propagating flame with the multicomponent model, with the mixture-averaged model, and in the planar flame. Thus the driving force of the Soret effect is 1.2 times larger than would be anticipated from the temperatures predicted by the mixture-averaged model.

\subsection{Markstein Relation Discrepancy}

Naturally propagating, premixed, lean, hydrogen-air flames show a discrepancy in the Markstein relation for the local flame speed that is exacerbated by the Soret effect. Asymptotic analysis (see, for example [36, p. 95]) predicts that curvature and strain modulate the local flame speed through an expression of the form

$$
s=s_{0}-s_{0} \mathcal{L} \kappa-\mathcal{L} S .
$$

This relation was verified experimentally by Aung et al. [37] for smoothly-expanding, spherical flames over a range of lean and rich hydrogen-air mixtures. The experimental results for $\phi<0.6$ were judged provisional because of the rapid onset of cellular structures.

An indication of this relationship in the cellular flame is given by the JPDF of $s$ and $\kappa$ in Fig. 6. In both cases the distributions have some trend lines placing $s>s_{0}$ even when $\kappa=0$ (more so with the multicomponent model). An investigating of the flames (not shown) indicates these trends occur where a flame cell briefly exhibits negative curvature prior to local extinction (which results in cell division). Such an event is occurring, for example, in Fig. 2 (top, left).

The salient feature of the theory is that Eqn. (8) is a first order linear model to predict $s$ in terms of the variables $\kappa$ and $S$, and the three unknown coefficients $c_{0}=s_{0}, c_{1}=s_{0} \mathcal{L}$, and $c_{2}=\mathcal{L}$,

$$
s=c_{0}-c_{1} \kappa-c_{2} S
$$

The values of the coefficients can be determined from laboratory observations of laminar flames as was done by Aung et al. Alternatively, for unstable flames, the coefficients can be determined by statistical estimation from direct 
numerical simulations. The Gauss-Markov theorem says the method of least squares can be used to calculate the maximum-likelihood estimate of the coefficients $c_{0}, c_{1}, c_{2}$ from sampled values for $s, \kappa, S$. We sample the values at uniformly spaced time steps and at uniformly spaced points along the $1144 \mathrm{~K}$ isotherms that correspond to the flame location. Since Fig. 2 shows that the flame extinguishes when the curvature is negative, sampling was restricted to regions where $\kappa \geq 0$ and $s \geq s_{0}$. Moreover, since the relevant tangential strain is the one upstream of the flame (see, for example [38]), the value of $S$ associated with a point on the $1144 \mathrm{~K}$ isotherm is found by tracing a path in the direction of the negative temperature gradient down to the $500 \mathrm{~K}$ isotherm where the $S$ is actually evaluated. In the asymptotic theory $c_{0} \equiv s_{0}$ and $c_{1} \equiv s_{0} c_{2}$, leaving just one free parameter, $\mathcal{L}$. Here, however, the relation $c_{3} \equiv s_{0} c_{2}$ is neglected, implicitly allowing different Markstein lengths for curvature and strain. Thus, the method of least squares is used to estimate the two numbers, $c_{1}$ and $c_{2}$, by minimizing the sum of squared residual errors, $\left[s-\left(s_{0}-c_{1} \kappa-c_{2} S\right)\right]^{2}$, over all the sampled values of $s, \kappa$, and $S$.

Table 1 reports the maximum likelihood values of the coefficients, scaled by $\delta_{T}$ and $s_{0}$. Fig. 7 shows the probability density of the normalized estimation error, $\left(s-s^{\text {est }}\right) / s_{0}$, where $s^{\text {est }}$ is determined from the general linear model, Eqn. (9), using the maximum likelihood coefficients in Tab. 1. The optimally chosen coefficients give the modeling error of smallest variance. No other coefficients can produce an error distribution with a smaller variance than the distribution Fig. 7. Overall, the linear Markstein relation of Eqn. (8) appears to be a poor model of the local flame speed in these naturally propagating, thermo-diffusively unstable flames. This conclusion does not contradict the work of Aung et al. [37], which was restricted to flames not exhibiting the cellular instability.

\subsection{Cellular Flame Morphology}

The mixture-averaged and multicomponent models predict flames of quite different dynamics and shapes. Since the local flame speed of the mixture-averaged model is lower, the multicomponent model has a more animated flame. A cell division or extinction occurs on average every $5.0 \mathrm{~ms}$ with the multicomponent model, but only every 11.2 ms with the mixture-averaged model. The ratio much exceeds the reciprocal ratio of average flame speeds. Thus the 
multicomponent model produces a different dynamic than merely speeding up the flame. The flame cells are smaller and more sharply curved, as shown by the distribution of curvature in Fig. 8.

\section{Conclusions}

Several differences are apparent between the multicomponent transport model, with Dufour and Soret effects, and the mixture-averaged approximation without cross-diffusion, in the present two-dimensional simulations of naturally propagating, lean, premixed, hydrogen-air flames:

- The propagation speed of the entire flame brush with the mixture-averaged model is $18 \%$ less than the speed predicted by the multicomponent model. There is greater reduction locally, being about $33 \%$ based on the modes of the distributions of the local flame speeds.

- Thermo-diffusively unstable flames have enhanced diffusion of fuel to positively curved flame surfaces. Based on the effective fuel equivalence ratio, the mixture-averaged model delivers only $57 \%$ of the fuel enrichment compared to the multicomponent model. Note, the reaction paths do not change other than proportionally with fuel consumption.

- Both the peak temperature and the driving term of the Soret effect, $|\nabla T| / T$, are larger with the multicomponent model than would be anticipated from the mixture-averaged model.

- The linear Markstein model, even with maximum likelihood estimates for coefficients, gives poor predictions of the local flame speed in these cellular flames.

- The cellular structures of the thermo-diffusively unstable flame are larger and less likely to extinguish or to divide with the mixture-averaged model than with the multicomponent model.

These differences for naturally propagating flames demonstrate that the impact of multicomponent transport is greater than was indicated by studies of planar and conical flames, $[12,16]$. Moreover, this work underscores 
the need to incorporate multicomponent transport in new simulations. It may be necessary to reexamine conclusions about premixed, lean, hydrogen-air flames that have been drawn from direct numerical simulations using simplified transport models.

\section{Acknowledgments}

This work was supported through the SciDAC program by the Director, Office of Science, Office of Advanced Scientific Computing, of the U.S. Department of Energy under Contract No. DE-AC02-05CH11231.

\section{References}

[1] P. Chiesa, G. Lozza, L. Mazzocchi, J. Eng. Gas Turbines Power 127 (2005) 73-80.

[2] W. Breitung et al., Nuclear Engineering and Design 253 (2005) 253-270.

[3] R. W. Shefer, Int. J. Hydrogen Energ. 28 (2003) 1131-1141.

[4] G. H. Markstein (Ed.), Nonsteady Flame Propagation, Pergamon Press, Oxford, 1964.

[5] K. T. Aung, M. I. Hassan, G. M. Faeth, Combust. Flame 109 (1997) 1-24.

[6] Y. Dong, A. T. Holley, M. G. Andac, F. N. Egolfopoulos, S. G. Davis, P. Middha, H. Wang, Combus. Flame 142 (4) (2005) 374-387.

[7] F. N. Egolfopoulos, C. K. Law, Proc. Combust. Inst. 23 (1990) 333-340.

[8] N. J. Brown, K. L. Revzan, Int. J. Chem. Kinet. 37 (2005) 538-553.

[9] M. K. Mishra, R. Yetter, Y. Reuvan, H. Rabitz, M. D. Smooke, Int. J. Chem. Kinet. 26 (1994) 437-443.

[10] M. D. Smooke, H. Rabitz, Y. Reuven, F. L. Dryer, Combust. Sci. Technol. 59 (1988) 295-319. 
[11] B. A. Williams, Combust. Flame 124 (1-2) (2001) 330-333.

[12] H. Bongers, L. P. H. de Goey, Combust. Sci. Technol. 175 (2003) 1915-1928.

[13] J. B. Greenberg, Combust. Sci. Technol. 24 (1) (1980) 83-88.

[14] C. T. Bowman et al., GRI-Mech 2.11, available at http://www.me.berkeley.edu/gri_mech.

[15] R. J. Kee, J. F. Grcar, M. D. Smooke, J. A. Miller, PREMIX: A Fortran Program for Modeling Steady, Laminar, One-Dimensional Premixed Flames, Technical Report SAND85-8240, Sandia National Laboratories, Livermore (1983).

[16] A. Ern, V. Giovangigli, Combust. Theory Modelling 2 (1998) 349-372.

[17] M. Baum, T. J. Poinsot, D. C. Haworth, N. Darabiha, J. Fluid Mech. 281 (1994) 1-32.

[18] J. B. Bell, R. K. Cheng, M. S. Day, I. G. Shepherd, Proc. Combust. Inst. 31 (2007) 1309-1317.

[19] H. G. Im, J. H. Chen, Combust. Flame 131 (2002) 246-258.

[20] G. Patnaik, K. Kailasanath, K. J. Laskey, E. S. Oran, Proc. Combust. Inst. 22 (1989) 1517-1526.

[21] M. Tanahashi, M. Fujimura, T. Miyauchi, Proc. Combust. Inst. 28 (2000) 529-535.

[22] M. Tanahashi, Y. Nada, Y. Ito, T. Miyauchi, Proc. Combust. Inst. 29 (2002) 2041-2049.

[23] T. Mitani, F. A. Williams, Combust. Flame 39 (1980) 169-190.

[24] V. Giovangigli, Multicomponent Flow Modeling, Birkhäuser, Boston, 1999.

[25] L. M. T. Somers, The Simultation of Flat Flames with Detailed and Reduced Chemical Models, Ph.D. thesis, Eindhoven University of Technology, available at http://yp.wtb.tue.nl/pdfs / 1684 .pdf (1994).

[26] G. Dixon-Lewis, G. L. Isles, R. Walmsley, Proc. Roy. Soc. London Ser. A 331 (1973) 571-584. 
[27] J. Warnatz, Ber. Bunsenges. Phys. Chem. 82 (1978) 643-649.

[28] D. E. Rosner, R. S. Israel, B. La Mantia, Combust. Flame 123 (2000) 547-560.

[29] M. S. Day, J. B. Bell, Combust. Theory Modelling 4 (2000) 535-556.

[30] A. Ern, V. Giovangigli, Multicomponent Transport Algorithms, Vol. m24 of Lecture Notes in Physics, SpringerVerlag, Berlin, 1994.

[31] A. Ern, V. Giovangigli, Phys. rev. E 53 (1996) 485-492.

[32] J. B. Bell, M. S. Day, J. F. Grcar, M. J. Lijewski, Comm. App. Math. Comput. Sci. 1 (1) (2005) $29-52$.

[33] G. I. Sivashinsky, Annual Review of Fluid Mechanics 15 (1983) 179-200.

[34] Y. B. Zeldovich, Theory of Combustion and Detonation in Gases (in Russian), Acad. Sci. USSR, 1944.

[35] E. L. Korn, B. I. Graubard, The American Statistician 52 (1) (1998) 58-69.

[36] N. Peters, Turbulent Combustion, Cambridge University Press, Cambridge, 2000.

[37] K. T. Aung, M. I. Hassan, G. M. Faeth, Combust. Flame 112 (1998) 1-15.

[38] B. Deshaires, P. Cambray, Combust. Flame 82 (1990) 361-375.

[39] S. G. Davis, A. V. Joshi, H. Wang, F. N. Egolfopoulos, Proc. Combust. Inst. 30 (2005) 1283-1292.

[40] P. Glarborg, M. U. Alzueta, K. Dam-Johansen, J. A. Miller, Combust. Flame 115 (1998) 1-27.

[41] G. P. Smith et al., GRI-Mech 3.0, available at http://www.me.berkeley.edu/gri_mech.

[42] S. C. Li, P. A. Libby, F. A. Williams, Proc. Combust. Inst. 24 (1992) 1503-1512.

[43] J. A. Miller, Chemical mechanism for methane combustion, privately distributed. 
[44] M. O’Connaire, H. J. Curran, J. M. Simmie, W. J. Pitz, C. K. Westbrook, Int. J. Chem. Kinet. 36 (2004) $603-622$.

[45] D. R. Dowdy, D. B. Smith, S. C. Taylor, A. Williams, Proc. Combust. Inst. 23 (1990) 325-332.

[46] S. D. Tse, D. L. Zhu, C. K. Law, Proc. Combust. Inst. 28 (2000) 1793-1800.

[47] C. Vagelopoulos, F. Egolfopoulos, C. Law, Proc. Combust. Inst. 25 (1994) 1341. 
Table 1: Maximum likelihood estimates of the coefficients in the general linear model for local flame speed. Normalization factors are $\delta_{T}=789 \mu$ and $s_{0}=15.52 \mathrm{~cm} / \mathrm{s}$.

\begin{tabular}{|c|c|c|c|}
\hline model & $c_{0} / s_{0}$ & $c_{1} /\left(\delta_{T} s_{0}\right)$ & $c_{2} / \delta_{T}$ \\
\hline mixture-averaged & $\equiv 1$ & -1.232 & -0.107 \\
multicomponent & $\equiv 1$ & -1.406 & -0.098 \\
\hline
\end{tabular}




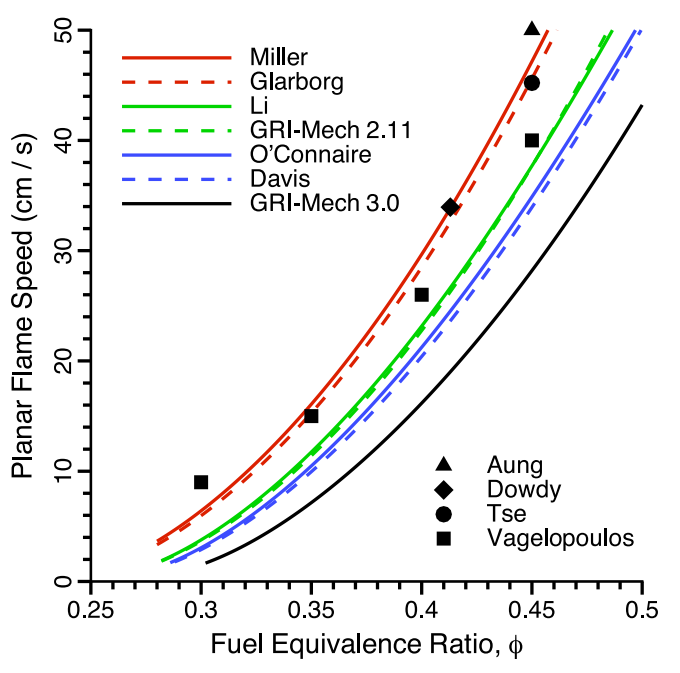

Figure 1: (lines) Predicted and (symbols) measured speeds of planar, premixed, lean, hydrogen-air flames. Predictions are from the CHEMKIN-PREMIX program [15] using its mixture-averaged transport model. The chemical mechanisms are: Davis [39], Glarborg [40], GRI-Mech 2.11 and 3.0 [14, 41], Li [42], Miller [43], and O'Connaire [44]. Measured data is from: Aung [37], Dowdy [45], Tse [46], and Vagelopoulos [47]. For these calculations, the premixed gas consists only of $\mathrm{H}_{2}, \mathrm{O}_{2}, \mathrm{~N}_{2}$ and all nitrogen species except $\mathrm{N}_{2}$ are deleted from the mechanisms. 

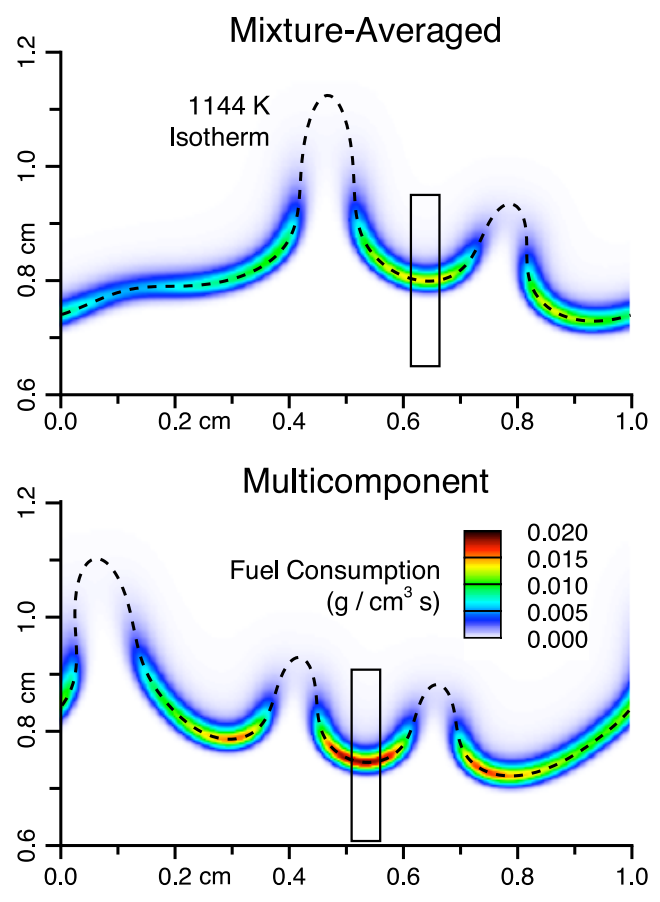

Figure 2: Representative images of fuel consumption in the 2D calculations. In the frame of reference of these figures, the flames burn downward and the gas flows upward.

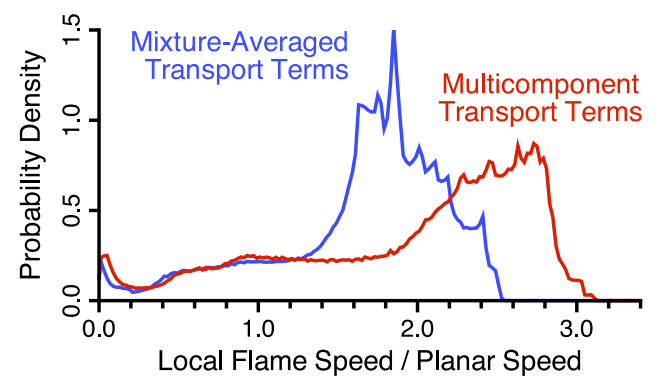

Figure 3: Probability densities of local flame speed in the 2D calculations relative to the planar (flat) flame speed, and conditioned by positive curvature. 


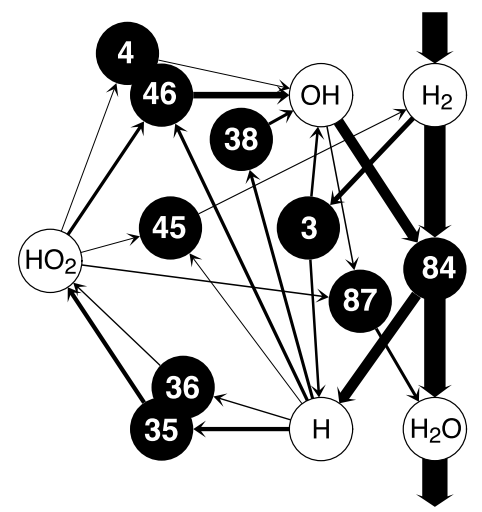

\begin{tabular}{|c|c|c|c|c|}
\hline \multicolumn{5}{|c|}{ integrated reaction rate of progress (normalized to $\max =1$ ) } \\
\hline \multicolumn{3}{|c|}{ GRI-Mech 2.11 number and reaction } & multi. & m.-av. \\
\hline 84. & $\mathrm{OH}+\mathrm{H}_{2}=$ & $=\mathrm{H}+\mathrm{H}_{2} \mathrm{O}$ & 1.000 & 1.000 \\
\hline 35. & $\mathrm{H}+\mathrm{O}_{2}+\mathrm{H}_{2} \mathrm{O}=$ & $=\mathrm{HO}_{2}+\mathrm{H}_{2} \mathrm{O}$ & 0.400 & 0.404 \\
\hline 46. & $\mathrm{H}+\mathrm{HO}_{2}=$ & $=2 \mathrm{OH}$ & 0.306 & 0.299 \\
\hline 38. & $\mathrm{H}+\mathrm{O}_{2}=$ & $=\mathrm{O}+\mathrm{OH}$ & 0.286 & 0.290 \\
\hline 3. & $\mathrm{O}+\mathrm{H}_{2}=$ & $=\mathrm{H}+\mathrm{OH}$ & 0.237 & 0.230 \\
\hline 87. & $\mathrm{OH}+\mathrm{HO}_{2}=$ & $=\mathrm{O}_{2}+\mathrm{H}_{2} \mathrm{O}$ & 0.142 & 0.148 \\
\hline 36. & $\mathrm{H}+\mathrm{O}_{2}+\mathrm{N}_{2}=$ & $=\mathrm{HO}_{2}+\mathrm{N}_{2}$ & 0.128 & 0.127 \\
\hline 4. & $\mathrm{O}+\mathrm{HO}_{2}=$ & $=\mathrm{OH}+\mathrm{O}_{2}$ & 0.060 & 0.063 \\
\hline 45. & $\mathrm{H}+\mathrm{HO}_{2}=$ & $=\mathrm{O}_{2}+\mathrm{H}_{2}$ & 0.052 & 0.051 \\
\hline
\end{tabular}

Figure 4: Integrated reaction network for hydrogen in the 2D calculations. The thickness of an arrow corresponds to the moles of hydrogen atoms exchanged. All paths that are at least $2 \%$ of the thickest are shown. The individual reactions are shown on the right with the original GRI-Mech 2.11 numbering and the integrated reaction rates of progress.
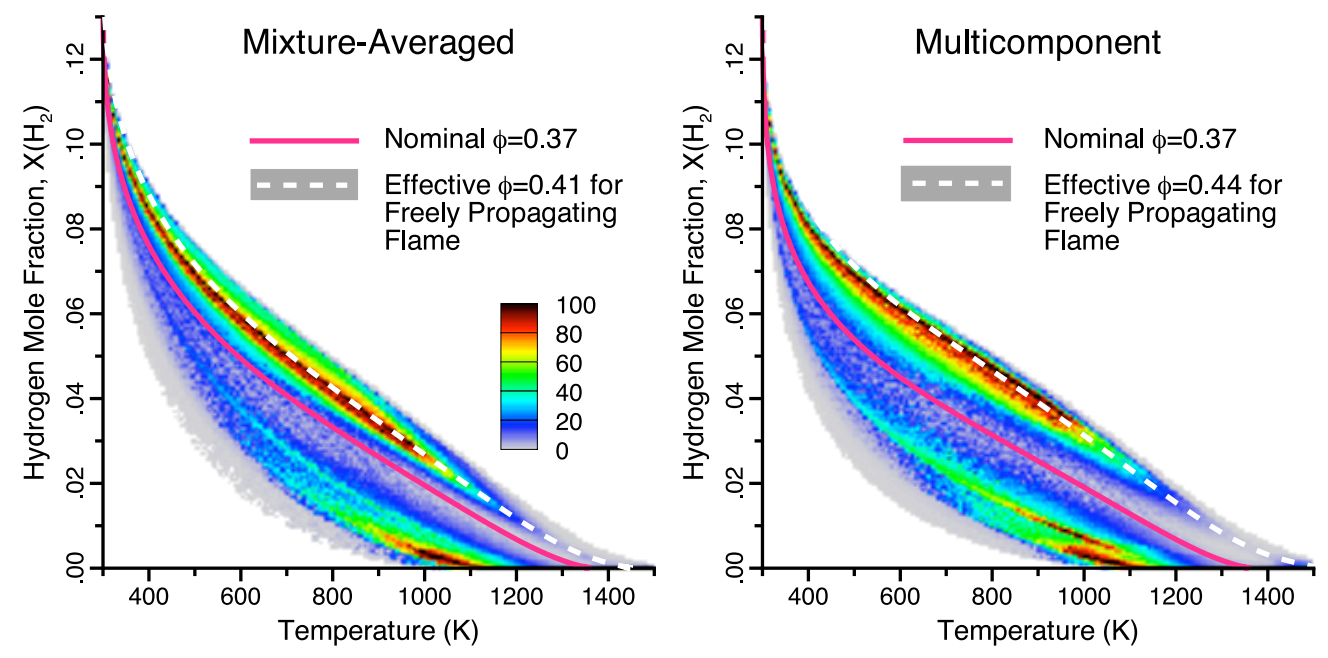

Figure 5: JPDF of fuel and temperature in the freely propagating flame represented by the method of conditional percentiles [35]. The superimposed solid and dashed lines are distributions for planar flames of various $\phi$. 

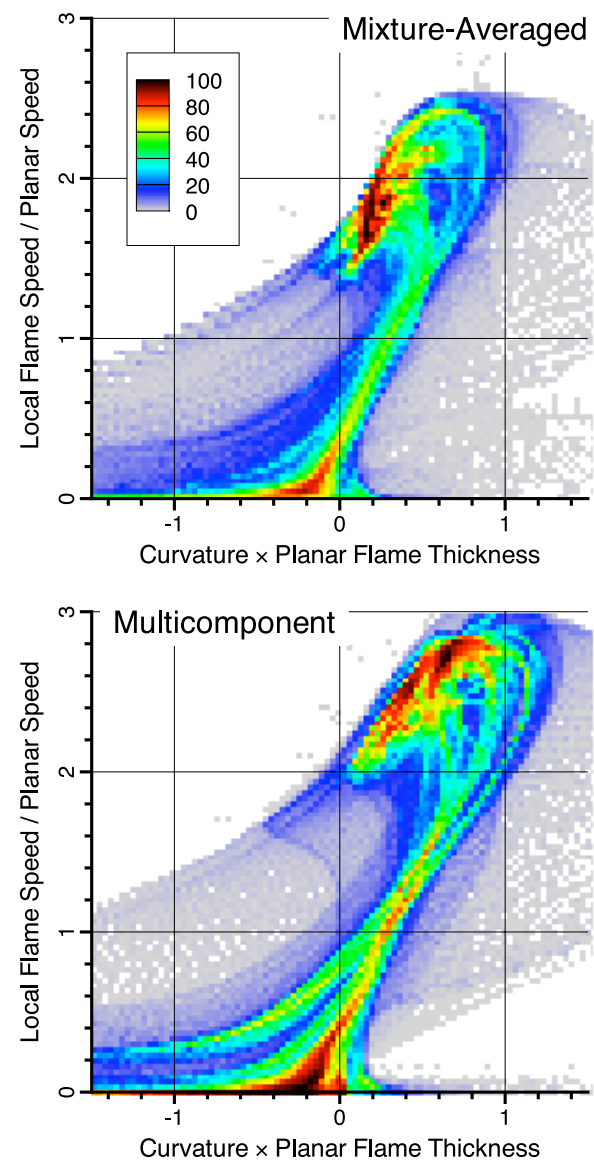

Figure 6: JPDF of curvature and local flame speed for the flame simulations with the mixture-averaged and multicomponent transport models. The percentiles of the distribution are shown: for example, the densest region that comprises $20 \%$ of the total probability is colored red and black.

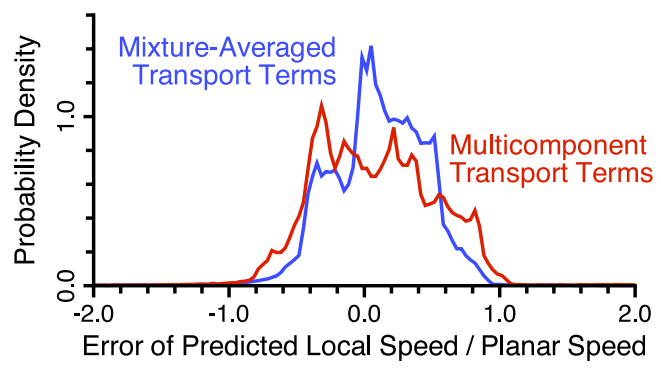

Figure 7: Probability densities of error in the general linear (Markstein) model for local flame speed. The coefficients used in Eqn. (9) are given in Tab. 1. 


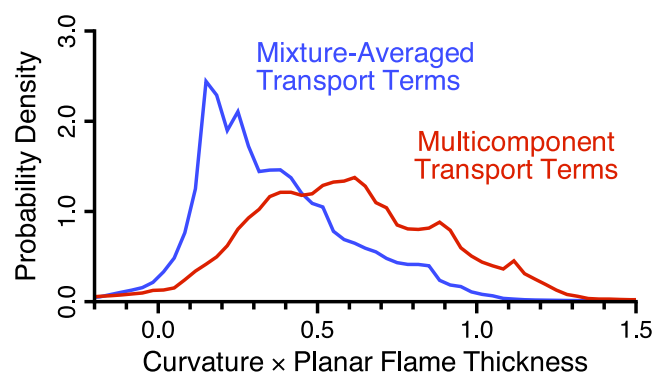

Figure 8: Probability densities of flame curvature obtained with the mixture-averaged and multicomponent transport models. The curvature is actually that of the 1144 isotherm which serves to mark the flame location. Since potions of the isotherm pass through burned regions, as shown in Fig. 2, curvature is sampled only where $s \geq s_{0}$. 